\title{
AVANCE DE RESULTADOS DE LOS NUEVOS TRABAJOS ARQueOlÓGICOS EN CUEVA DE LA COCINA (Dos Aguas, ValenCIA): CAMPAÑA DE 2015
}

\section{ANTECEDENTES}

Durante tres semanas (del 15 de junio al 5 de julio de 2015) se ha llevado a cabo una campaña de excavación sistemática en Cueva de la Cocina (Dos Aguas, Valencia) que ha consistido en la realización de una serie de sondeos en el interior de esta gran cavidad. El yacimiento fue objeto de varias intervenciones arqueológicas en el pasado siglo. En los años 40 (entre los años 1941 y 1945) Lluís Pericot dirigió los trabajos de campo en el área de la entrada (suroriental) dónde efectuó 4 grandes sondeos contiguos que supusieron la excavación de una extensión aproximada de unos $85 \mathrm{~m}^{2}$. En el año 1945 se publicó uno de los trabajos de referencia sobre este yacimiento (Pericot 1945) en el cual se daba a conocer una interesante secuencia con ocupaciones del Mesolítico y Neolítico basada en el análisis de la intervención efectuada ese mismo año. Sin duda, el trabajo de Javier Fortea (1973) resultó clave para entender la secuencia de Cocina. Este autor estudió los materiales líticos correspondientes a este mismo sector excavado en el año 1945 y estableció una secuencia en cuatro fases que, se suponía, englobaba el desarrollo de los últimos caza-recolectores (fases A y B) y la neolitización de éstos (fases C y D). Cocina se convirtió en el yacimiento príncipe de la secuencia epipaleolítica, de raiz tardenoide, en el Mediterráneo peninsular (Fortea 1973; Fortea et al. 1987; Juan Cabanilles y García Puchol 2013; Martí et al. 2009). Entre los años 1974 y 1981 Javier Fortea dirigió un total de 8 campañas de excavación en el lugar, de las cuales únicamente se han publicado algunos resultados preliminares (Fortea 1975; Fortea et al. 1987; Fumanal 1986). Fortea intervino en el interior de la cavidad, en una área extensa situada al noroeste de la intervención anterior, además de efectuar algunos sondeos en diferentes puntos de la misma. En el marco del proyecto HAR 2012-33111 "Meso Cocina: Los últimos caza-recolectores y el paradigma de la neolitización en el mediterráneo peninsular" hemos procedido al análisis integro de las campañas de Pericot y Fortea teniendo en cuenta que la mayoría de la información resultaba inédita hasta la fecha. Se está realizando de este modo el análisis de los materiales depositados en 


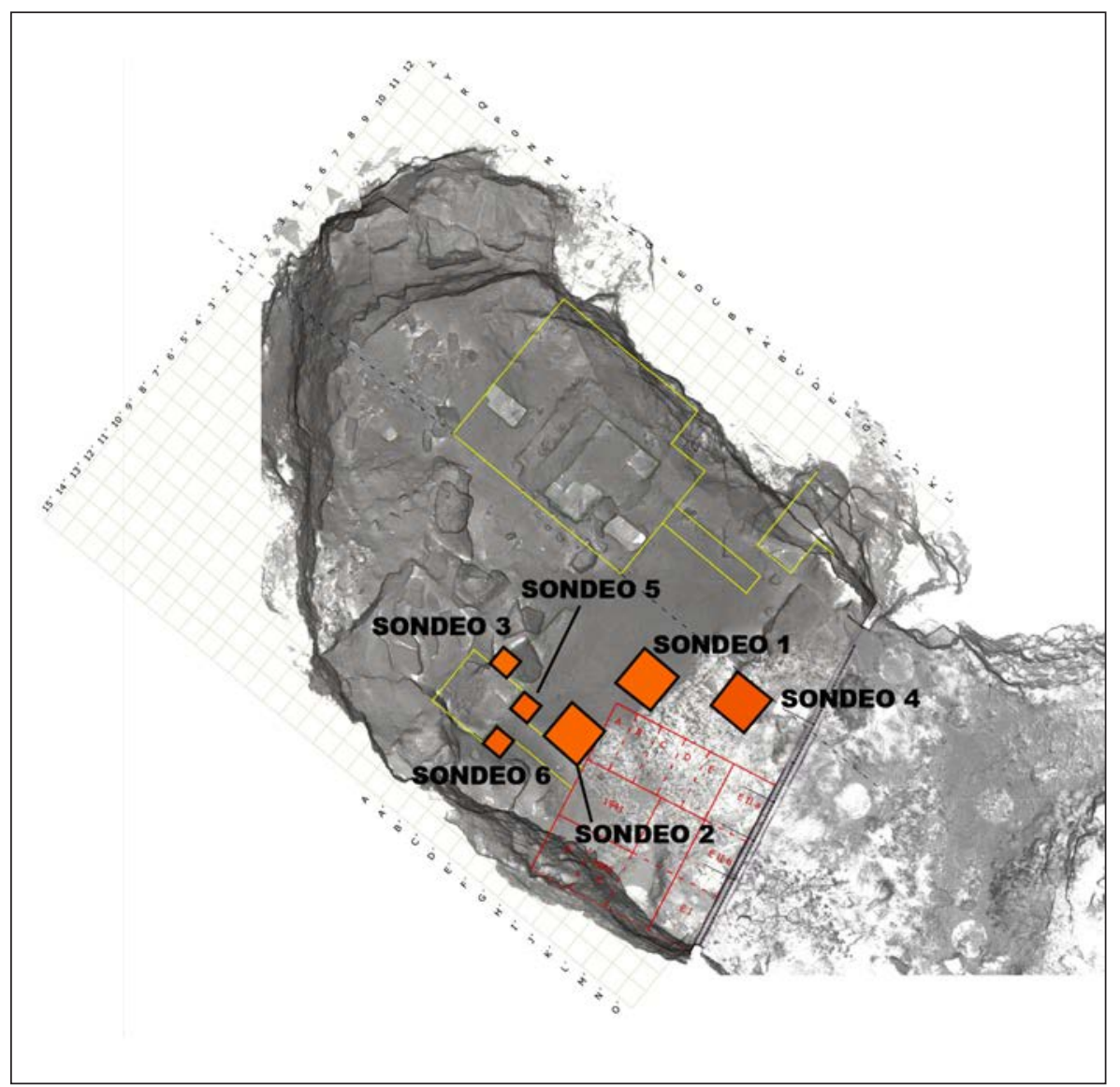

Fig. 1. Planta de la cavidad con indicación de los sondeos efectuados en esta campaña ( 1 a 6 ) En color amarillo los sondeos efectuados por Fortea, en rojo el área de excavación de Pericot. Podemos ver la proyección de la cuadrícula $\left(1 \mathrm{~m}^{2}\right)$ establecida por Fortea y que ha servido de base para los trabajos actuales.

el Museo de Prehistoria de Valencia a la vez que tratamos de reconstruir la secuencia en el lugar a partir de toda la información existente (diarios de campo, documentación planimétrica, cortes, material fotográfico: García Puchol et al. 2014; Pascual Benito y García Puchol 2015). La actuación que describimos a continuación ha tenido como objetivo principal el esclarecimiento de cuestiones planteadas durante el proceso de estudio en el laboratorio.

\section{METODOLOGÍA}

En el desarrollo de la intervención se ha seguido el sistema Harris adaptado a una excavación en cueva de forma que se han asignado UUEE de relleno de una profundidad máxima de $5 \mathrm{~cm}$. o bien UUEE negativas. Todas las UUEE han quedado descritas en una ficha Harris diseñada para tal fin. Se han llevado a cabo diferentes sondeos y muestreos en diferentes puntos de la cavidad. La información espacial ha sido documentada mediante la utilización de una estación total que ha permitido georeferenciar todos los datos. Previamente a la actuación descrita llevamos a cabo el escaneado 3D de la cavidad, con la estructura metálica de la excavación en extensión de Fortea que ha servido de base para situar los sondeos de Pericot (fig. 1). Decidimos seguir la cuadrícula de Fortea aunque añadiendo a su denominación un distintivo que a nuestro entender facilita la diferenciación de los cuadros (eliminando las comas altas: A', B', C', etc.). Dado la situación de los sondeos practicados, las letras añadidas han sido $\mathrm{S}$ y E (sur y este). Los cuadros están subdivididos a su vez en subcuadros de 0,5 metros referidos con un número. Se ha llevado a cabo una exhaustiva toma fotográfica del levantamiento de cada una de las UUEE, rectificándose las mismas para obtener un dibujo de la planta cuando se ha considerado necesario. Del mismo modo se han tomado muestras de todas las UUEE de relleno (unos 5 litros) para su tamizado con agua. Si los hallazgos lo requerían se han tomado muestras completas de sedimento. El material significativo ha sido situado según sus coordenadas geográficas. 


\section{DESARROLLO DE LA ACTUACIÓN ARQUEO- LÓGICA}

Nuestro objetivo con esta intervención ha sido recabar información estratigráfica con el fin de reconstruir la secuencia del yacimiento. La información relativa a la estratigrafía de las excavaciones de Pericot era escasa, algunas breves anotaciones sobre la coloración del sedimento y la presencia de grandes bloques, además de los dibujos esquemáticos de algunos cortes. De las excavaciones de Fortea disponemos en cambio de datos pormenorizados sobre las características estratigráficas. Nos centramos en un primer momento en el sector de Pericot, dado que la documentación disponible era menor. Tratamos para ello de encontrar los cortes de la campaña de 1943, para lo cual disponíamos de su dibujo, además de que la planimetría de Fortea reflejaba sus límites (fig. 2). Tras el replanteo de su situación en planta procedimos a vaciar una parte del sondeo de Pericot, dado que dicha área se había rellenado parcialmente de tierras, abarcando para ello una superficie extensa que trataba de delimitar el corte norte de 1943. Al mismo tiempo abrimos un primer sondeo de $4 \mathrm{~m}^{2}$ justo fuera de este límite (cuadros FE 3S, GE 3S, FE 4S, GE 4S). A partir de este sondeo (sondeo 1) pudimos seguir el corte de Pericot (cuadros FE 4S, GE 4S). Desde el interior de la cata de Pericot seguimos el corte del año 43 que presenta una superficie irregular debido a su desmoronamiento. En este punto realizamos un corte recto del mismo donde podimos observar que presenta un sedimento rojo estéril con una serie de laminaciones en dirección contraria al buzamiento arqueológico descrito por su excavador. Coincide el tramo de corte expuesto ahora con el dibujo que presenta Pericot del corte de este año, lo que sirve para deducir que en este punto estamos ya en el nivel rojo estéril de la cavidad, que fue excavado por Pericot atendiendo a las capas artificiales consideradas anotando que hacia el extremo norte apenas aparecía material arqueológico. Buena parte del corte del año 43 reflejaba por tanto el nivel de arcillas rojas estéril descrito por Pericot, lo cual viene a confirmar la presencia de una hondonada al sur de la cavidad, en la zona de la entrada, que albergaría el grueso del relleno prehistórico. Al mismo tiempo nos propusimos vaciar una trinchera excavada en los años 70 por Fortea y que unía la cata de Pericot (sondeo 1941) con un sondeo realizado por él hacia el interior de la cavidad (cuadros A' 8, A' 9, A' 10, B' 8, B' 9, B' 10 de Fortea) -fig. 3-. El vaciado de la misma resultó sencillo dado que estaba perfectamente delimitada en planta y los perfiles dejados eran fáciles de

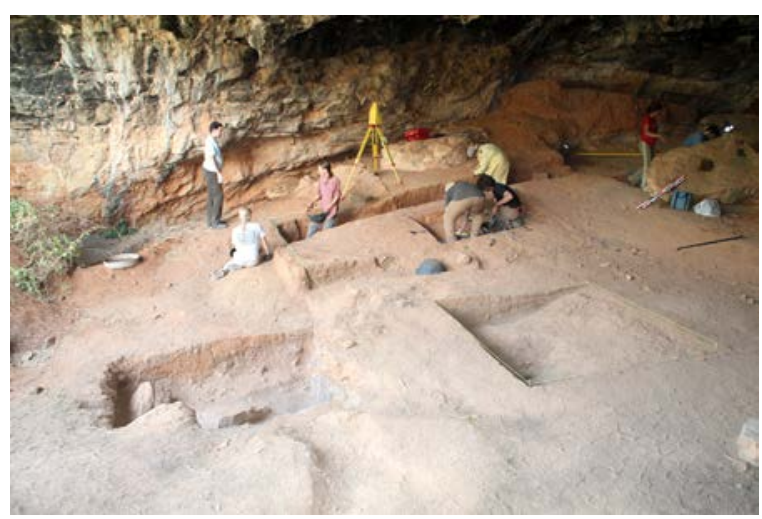

Fig. 2. Panorámica general de los sondeos efectuados en la reciente campaña de 2015.

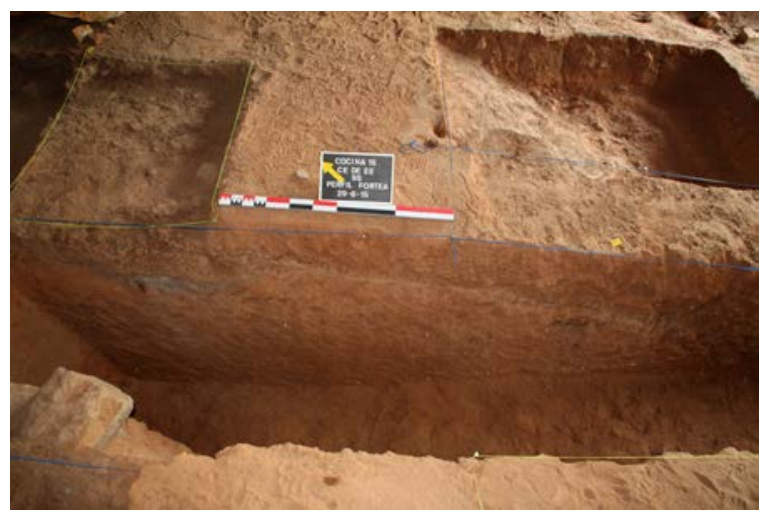

Fig. 3. Foto de detalle de los sondeos 2 y 5 desde la zanja efectuada por Fortea.

seguir (muestreos 1, 2 y 3). En este punto planteamos un segundo sondeo que trataba de delimitar el corte transversal de la cata de Pericot y cuya estratigrafía podíamos seguir desde el vaciado de la zanja. El sondeo 2 ha abarcado los cuadros EE 7S, FE 7S, EE 8S, FE 8S. Un tercer sondeo se ha llevado a cabo en el sector pequeño excavado por Fortea (cuadros A' 8, A' 9, A' 10, B' 8, B' 9, B' 10). En este punto nos limitamos a limpiar un área de $6 \mathrm{~m}^{2} \mathrm{y}$ a practicar un pequeño sondeo (sondeo 3 ) en el cuadro A'7'de Fortea (AE 7S) siguiendo el perfil dejado por la excavación de éste una vez se hubo procedido a su limpieza. Un cuarto sondeo (sondeo 4) se ha practicado en el área de la entrada de la cueva (cuadros JE 1S, JE 2S, KE 1S, KE 2S) con el fin de comprobar si quedaba depósito intacto en este punto. El sondeo 5 se localiza en los cuadros CE 7S y CE 8S. Por último, el sondeo 6 corresponde a una cata de $1 \mathrm{~m}^{2}$ realizada en el cuadro CE $10 \mathrm{~S}$. 
La profundidad alcanzada en los diferentes sectores ha sido desigual, bien hasta alcanzar el nivel rojo estéril o bien dejando en planta nivel fértil. En relación con dos de los sondeos excavados (sondeo 2 y 5 ) se han llevado a cabo muestreos hasta alcánzar las arcillas estériles (muestreo 1, 2 y 3). En todos los casos los muestreos se han practicado a partir de la zanja de Fortea, atendiendo a capas artificiales de un máximo de $5 \mathrm{~cm}$. El muestreo 1 corresponde al cuadro CE-8S (subcuadro 4). El muestreo 2 se sitúa en el cuadro EE-8S (subcuadros 3 y 4). Se practicó un tercer muestreo de $10 * 10 \mathrm{~cm}$ (muestreo 3) situado entre ambos con el fin de obtener una columna de sedimento.

\section{CONCLUSIÓN}

Los trabajos desarrollados durante esta campaña de campo en Cueva de la Cocina han contribuido a la mejor comprensión de la secuencia prehistórica en el lugar a través de la realización de toda una serie de sondeos alrededor de la gran cata practicada por Pericot en los años $40 \mathrm{y}$ de algunos de los sondeos efectuados por Fortea ya en los años 70/80. Nuestro principal objetivo residía precisamente en la localización de depósito intactos en el área inmediata a estos trabajos con el fin de obtener un muestreo actual que permita la comprensión de la estratigrafía En el marco del proyecto HAR 2012-33111 estamos llevando a cabo la revisión de los trabajos de actuación previos en la cavidad con el fin de proceder a su completa publicación y relectura a través de un análisis actualizado. La intervención realizada ha posibilitado la exposición parcial del corte de Pericot y su seguimiento desigual a partir de los cálculos efectuados teniendo en cuenta la planimetría de Fortea. La exposición parcial del mismo permitió su refresco y la comprobación del dibujo efectuado en su día por Pericot. La conservación desigual del depósito en la cavidad ha quedado patente. A medida que nos adentramos hacia el interior los depósitos arqueológicos reducen su espesor, de hecho parece corroborado como buena parte del depósito neolítico y posterior apenas se conservaba, exceptuando determinados puntos, al menos en el momento de la intervención de Fortea. En la actualidad parecen advertirse indicios de una mejor conservación del depósito neolítico hacia el interior de la cavidad, junto a la pared derecha, en un punto donde el depósito alcanza su cota más alta. En este lugar ampliamos uno de los cortes de Fortea en un espacio reducido de $0,5 \mathrm{~m}^{2}$ (sondeo 3 ) en el que comprobamos la aparición de cerámica prehistórica que podría corresponder a momentos del Neolítico antiguo/medio en la cavidad. El resto de sondeos practicados evidencian que hacia el exterior de la cavidad estos niveles no se conservan. Además no parece haber una solución de continuidad clara entre el sondeo inmediato practicado por nosotros y la cata de Fortea. En su lugar, siguiendo la zanja efectuada por Fortea hasta enlazar con el sondeo de Pericot, observamos una secuencia prehistórica muy corta (en comparación con la secuencia descrita por Pericot) y que englobaría un nivel prehistórico con un reducido número de fragmentos cerámicos y escasa fauna que se superpondría a un nivel de escasa potencia sin cerámicas, con gran concentración de cenizas, entre cuyos materiales destacan los restos líticos y la fauna. Una hipótesis a contrastar sería la existencia de una cicatriz erosiva que afectara a la secuencia mesolítica, aspecto que deberemos corroborar mediante el programa de dataciones radiocarbónicas previsto.

La conservación de depósito intacto que alberga mayores expectativas de extensión en profundidad estaría justo a la entrada de la cavidad (sondeo 4). En este punto, debajo de los restos del muro de cierre antiguo se ha conservado un rico nivel de materiales que desde techo evidencian su adscripción mesolítica.

Toda la información aquí expuesta y sobre todo el procesado de la misma que se está llevando a cabo en el laboratorio aportan nuevos datos que resultan del máximo interés para comprender la relevancia de la secuencia de Cocina en el marco del desarrollo del proceso de neolitización en el Mediterráneo peninsular.

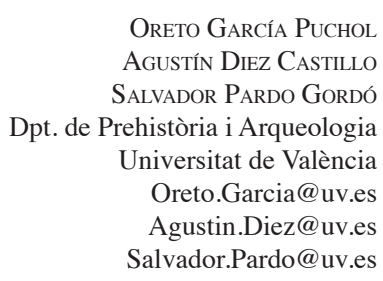

JOAQUim JuAN CABANILLES Museu de Prehistòria de València - S.I.P. Diputació Provincial de València joaquim.juan@dival.es

SARAH B. McCluRe Dpt. of Anthropology Pennsylvania State University Sbm19@psu.edu 


\section{AGRADECIMIENTOS}

Las investigaciones presentadas se han llevado a cabo en el marco del proyecto "MESO COCINA: los últimos caza-recolectores y el paradigma de la neolitización en el mediterráneo occidental" (HAR2012-33111), subvencionado por el Ministerio de Economía y Competividad, Gobierno de España.

Los trabajos de campo se han llevado a cabo dentro del programa de investigaciones del SIP-Museu de Prehistòria de la Diputación de Valencia.

\section{BIBLIOGRAFÍA}

FORTEA, J. (1973): Los complejos microlaminares y geométricos del Epipaleolítico mediterráneo español, Memorias del Seminario de Arqueología y Prehistoria 4, Salamanca.

FORTEA, J. 1975: En torno a la cronología relativa del inicio del arte levantino (Avance sobre las pinturas rupestres de la Cocina), Papeles del Laboratorio de Arqueología 11, 185-197.

FORTEA, J.; MARTÍ, B.; FUMANAL, P.; DUPRÉ, M. y PÉREZ RIPOLL, M. (1987): Epipaleolítico y neolitización en la zona oriental de la Península Ibérica, Premières Communautés Paysannes en Méditerranée Occidental. Actes du Colloque International du CNRS (Montpellier, 1983) (J. Guilaine, J: Courtin, J.-L. Roudil, J.-L. Vernet, dirs.), Paris, 599-606.

FUMANAL, M.P. (1986): Sedimentología y Clima en el País Valenciano, Serie Trabajos Varios del S.I.P. 83, Valencia.
GARCÍA PUCHOL, O.; JUAN CABANILLES, J.; McCLURE, S. B.; PASCUAL BENITO, J.LL.; MARTÍ, B.; PÉREZ RIPOLL, M.; BERNABEU, J.; PARDO, S.; MOLINA, LL.; CARRIÓN MARCO, Y.; DIEZ CASTILLO, A. (2014): Los últimos caza-recolectores en Cueva de la Cocina (Dos Aguas, Valencia), Los cazadores y recolectores del Pleistoceno y del Holoceno en Iberia y el estrecho de Gibraltar (R. Sala, coord.), Burgos, 370-372.

JUAN CABANILLES, J.; GARCÍA PUCHOL, O. (2013): Rupture et continuité dans la néolithisation du versant méditerranéen de la péninsule Ibérique: mise à l'épreuve du modèle de dualité culturelle, XXVIIe Congrès Préhistorique de France. Transitions, Ruptures et Continuité durant la Préhistoire/Transitions, Ruptures and Continuity in Prehistory (Bordeaux-Les Eyzies, 2010) (J. Jaubert, N. Fourment y P. Depaepe, eds.), Paris, 405-417.

MARTÍ, B.; AURA, J. E.; JUAN CABANILLES, J.; GARCÍA PUCHOL, O. Y FERNÁNDEZ, J. (2009): El mesolítico geométrico facies Cocina, El Mesolítico Geométrico en la Península Ibérica (Actas de la Reunión de Jaca, 2008) (P. Utrilla y L. Montes, coord.), Zaragoza, 205-258.

PASCUAL BENITO, J. LL.; GARCÍA PUCHOL, O. (2015): Los moluscos marinos del Mesolítico de la Cueva de la Cocina (Dos Aguas, Valencia), La Investigación Malacológica en la Península Ibérica: Nuevas aportaciones (I. Gutiérrez, D. Cuenca, M. R. González, eds), Santander, 65-75.

PERICOT, L. (1945): La Cueva de la Cocina (Dos Aguas). Nota preliminar, APL II, 39-71. 